\title{
El auge de la derecha en la crisis posneoliberal en América Latina: casos Brasil y Argentina*
}

\section{The rise of the right in the post- neoliberal crisis in Latin America: Brazil and Argentina}

\author{
José Arturo Tipismana Arriola ${ }^{\star \star}$ \\ Pontificia Universidad Católica del Perú \\ ORCID: 0000-0003-4217-5514
}

Fecha de recepción: 7 de enero del 2020

Fecha de aceptación: 28 de enero del 2020

\section{ISSN: 2415-2498}

Tipismana Arriola, J. A. (2019). El auge de la derecha en la crisis posneoliberal en América Latina: casos Brasil y Argentina. Politai: Revista de Ciencia Política, Año 10, segundo semestre, № 19, pp. 109-135.

DOI: https://doi.org/10.18800/politai.201902.005

\footnotetext{
* El autor agradece las invaluables contribuciones de Nicolás Jacobs, colaborador en la realización del presente trabajo de investigación.

** Estudiante de Ciencia Política y Gobierno de la Pontificia Universidad Católica del Perú (PUCP).
} 


\section{RESUMEN}

El presente artículo se centra en dos casos de estudio, Brasil y Argentina, en los que, tras varios años de gobiernos de izquierda, lograron triunfar y llegar a la presidencia dos candidatos de derecha. Estos son Jair Bolsonaro, quien fue candidato del Partido Social Liberal y actualmente es presidente de Brasil; Mauricio Macri, quien ganó la presidencia de la Argentina con la Coalición de derecha Cambiemos. Tomando en cuenta los resultados de las últimas elecciones presidenciales, parece ser que en Argentina este giro a la derecha no se pudo sostener en el tiempo; mientras que, en Brasil, el Partido de los Trabajadores no perdió su presencia significativa en el Parlamento. Esta investigación concluye que este cambio no sucedió debido a que, en principio, no se trató de un giro político similar al pink tide. Se toman en cuenta tres factores explicativos: el desempeño económico, la credibilidad de los líderes de izquierda y la fuerza de la derecha. A partir de estos se analizará cada caso a fondo, para luego compararlos y contrastarlos. El resultado de este proceso es la identificación de diferencias sustanciales, sobre todo en lo referido a la crisis económica y a la fuerza de la derecha. Finalmente, se presentan reflexiones de cómo estos resultados electorales solo representan el desgaste de los gobiernos de izquierda debido a los grandes escándalos de corrupción y al freno del crecimiento económico, y no necesariamente el inicio de una nueva era en la política latinoamericana.

Palabras clave: corrupción, legitimidad, giro a la izquierda, crecimiento económico, oposición.

\section{Abstract}

This paper focuses on two case studies, Brazil and Argentina, in which, after several years of leftist governments, two right-wing candidates succeeded and became president. These are Jair Bolsonaro, who was a candidate of the Social Liberal Party and is the current president of Brazil; and Mauricio Macri, who won the presidency of Argentina with the right-wing Coalition Cambiemos. Considering the results of the last presidential elections, it seems that in Argentina this right turn could not be sustained over time; meanwhile, in Brazil, the Partido de los Trabajadores achieve a relevant presence in Parliament. This paper concludes that this turn to the right did not happen because it was not a political turn like the pink tide. Three explanatory factors are considered: economic performance, the credibility of leftist leaders and the strength of the right. From there, each case will be thoroughly analyzed, and then compared. The result of this process is the finding of substantial differences, especially regards the economic crisis and the strength of the right. Finally, the reflections regarding the findings sustain that these electoral results only represent the attrition of leftist governments due to the great corruption scandals and the curb economic growth, and not necessarily the beginning of a new era in Latin American politics.

Keywords: corruption, legitimacy, turn to the left, economic grown, opposition. 


\section{INTRODUCCIÓN}

Actualmente existe una tendencia global sobre el incremento del apoyo a candidatos derecha radical. En Europa, movimientos conservadores de derecha están resurgiendo con una fuerza inusitada. En Francia, el Frente Nacional (Marine Le Pen) estuvo cerca de ganar las elecciones presidenciales del 2017; mientras que en República Checa, Libertad y Democracia Directa obtuvo excelentes resultados en las elecciones legislativas del 2017; en Austria, la coalición de gobierno actual incluye a la extrema derecha, pues están presentes el Partido del Pueblo, con Kurz de Canciller, y el Partido de la Libertad, con Stache de viceprimer ministro. Incluso en Alemania, especialmente en el Este, Alternativa por Alemania se ha fortalecido en detrimento del CDU y del SPD. Con esto se puede observar que el fenómeno a estudiar no es sólo de carácter regional, sino que podría ser parte de una nueva orientación política internacional. Sin embargo, la presente investigación se centrará en la región sudamericana debido a que esta vivió hace menos de veinte años un giro político completamente distinto: el giro a la izquierda

Por otro lado, los resultados electorales son indicadores de cómo la población de un país evalúa su situación y cuáles son las aspiraciones de cambio que tienen respecto al futuro, esto se desarrollará más adelante. La propuesta de esta investigación consiste en analizar los resultados electorales de dos países latinoamericanos con gran influencia regional, que han tenido elecciones presidenciales que los han encausado a destinos políticos que representan una ruptura con la tradición de izquierda de inicios del siglo XXI.

El trabajo se divide en cuatro apartados: el marco teórico, la revisión de literatura, el análisis de casos y las conclusiones. En el marco teórico, se realiza una breve discusión sobre la conceptualización de la dicotomía derecha-izquierda; además, se contextualizará el giro a la izquierda en América Latina y se presentarán teorías que sustentan la noción de giro político regional. En la revisión de literatura, se presentará información de los resultados electorales, las campañas políticas y las causas del giro a la izquierda, para luego dar paso al resurgimiento de la derecha, así como la importancia de los medios de comunicación, el financiamiento y los alcances de las campañas con miras a la elección. También se incluirán estudios sobre el posible giro a la derecha que está sucediendo en América Latina.

En el tercer apartado, se analizarán los casos de Brasil y Argentina, con base a criterios comunes justificados en la literatura; luego se realizará un contraste de ambos casos, buscando encontrar patrones comunes que contribuyan a la explicación de los resultados. En las conclusiones se recapitulará lo abordado en el trabajo, extrayendo reflexiones respecto al panorama político regional y la necesidad de investigar con mayor énfasis el tema aquí estudiado; así como también la idea de que los resultados electorales podrían no representar necesariamente el fin o inicio de una nueva época, sino más bien pueden ser productos del recambio.

La pregunta de investigación de este trabajo es la siguiente: ¿cuáles son los factores que explican la victoria electoral de la derecha en Argentina y Brasil? La 
respuesta es que existen tres factores que permiten explicar este supuesto giro: el desempeño económico; la credibilidad de los líderes de izquierda; y la fuerza de la derecha. El primer criterio suele ser un determinante clave en cuanto a la aprobación o desaprobación de un gobierno, al igual que la credibilidad de los líderes de este puede sumar o restar legitimidad. También es necesario considerar la fuerza de la oposición, en este caso la derecha, midiendo su capacidad de capitalizar el descontento de la población por medio de una alternativa creíble. En esa línea, la hipótesis es la siguiente: la combinación entre un deficiente desempeño económico, sumado a una mermada credibilidad de los líderes de izquierda y al fortalecimiento de la oposición, en los gobiernos del "giro a la izquierda", posibilitaron la victoria electoral de la derecha en Sudamérica.

Este tema es relevante debido a que, si bien el giro a la izquierda ha sido estudiado a profundidad por la academia, lo que parece ser el fin de este fenómeno no ha sido estudiado lo suficiente. Cuando se buscó bibliografía al respecto, se encontraron escasas fuentes, no obstante, se presentarán las más importantes. Los casos de Argentina y Brasil ameritan ser estudiados en el marco de este aparente giro debido a que son dos países con gran influencia geopolítica en la región, además de haber sido emblemas del pink tide. Estudiarlos permite tener un panorama general de lo que podría suceder en el resto de América Latina: su influencia fue un factor importante en la consolidación del giro a la izquierda.

La limitación central de nuestra investigación recae en el alcance explicativo que tiene que dos casos constituyan el inicio de un giro político regional, como el pink tide, pues si bien este trabajo parte de entender que el potencial giro a la derecha en Argentina y Brasil puede considerarse el inicio de un giro regional, esta afirmación no puede ser demostrada a cabalidad.

Leiras (2007), al referirse al giro a la izquierda, explica que definir un giro regional merece una amplia discusión al respecto; el autor cuestiona definir el giro a la izquierda como algo uniforme, consensuado o coordinado entre los países con gobiernos de izquierda. Además, menciona que el fracaso de las políticas neoliberales no implica per sé un giro. Trasladando la discusión al objeto de análisis del presente estudio, es necesario aclarar que se utiliza un concepto amplio de derecha dentro del cual existen variaciones en las características de los partidos de este corte ideológico que llegaron al poder, dependiendo de cada caso. Además, para evitar que el documento se base únicamente en la crisis del modelo previo, se está considerando como factor determinante la agencia de los partidos de derecha durante la crisis.

\section{MARCO TEÓRICO}

\subsection{Definiendo izquierda y derecha}

En el presente trabajo se utilizarán los términos "derecha" e "izquierda" para clasificar a candidatos, partidos y gobiernos. Estos términos son antitéticos, es decir, se contraponen. En ese sentido, Bobbio (1994) considera que "derecha" e "izquierda" son 
términos recíprocamente exclusivos y conjuntamente exhaustivos: exclusivos porque ninguna doctrina ni movimiento pueden ser a la vez de derecha y de izquierda; exhaustivos, porque una doctrina o movimiento únicamente puede ser de derecha o de izquierda.

Aunque esta dicotomía izquierda-derecha actualmente luce desgastada y se considera operativamente obsoleta debido a las variaciones y evoluciones en las últimas décadas, "la inercia fruto de dos siglos de vigencia explica que mucha gente siga recurriendo a esos conceptos para interpretar la acción política" (Navas 2014: 175). Esto quiere decir que, a pesar de que los movimientos, partidos y/o candidatos actuales no cumplen totalmente con las características tradicionalmente asignadas a ambos lados del espectro político, la división izquierda-derecha está vigente debido a que la población percibe que esta demarcación política aún es válida. Para fines de este trabajo se tomarán las definiciones generales y tradicionales de izquierda y derecha. Esto se debe básicamente a que como nuestro objeto de estudio son los resultados electorales, la percepción del ciudadano de a pie es particularmente importante.

El valor político fundamental que la izquierda defiende es la igualdad, mientras que la derecha se centra en la defensa de la libertad. En otras palabras "la izquierda busca la libertad a través de la igualdad; la derecha busca la igualdad a través de la libertad" (Navas 2014: 165). En términos económicos, "la izquierda confía en la planificación y regulación estatales (...) pone el acento en la distribución. El principio de reparto (...) sería la necesidad. La derecha confía más en el mercado y en la iniciativa privada. Prioriza la producción, la creación de riqueza. Su criterio de reparto sería el mérito" (Navas 2014: 166). No obstante, en especial desde un aspecto social, es importante remarcar que no toda derecha es liberal, sino que también puede ser conservadora.

El posicionamiento de la izquierda en América Latina se ha logrado a inicios del siglo XXI, asumiendo el poder. Estos líderes de izquierda se caracterizan por tener discurso populistas y de reivindicación, además, "se hizo más importante enfatizar nuevamente la oposición entre la izquierda y la derecha, o entre la socialdemocracia y el neoliberalismo, en lugar de insistir una vez más en el carácter distintivo de la centroizquierda contemporánea" (Noel y Therien, 2008: 189). Esta reafirmación de la división tradicional de izquierda-derecha implicaba también un retorno a la preocupación central de la política de izquierda-derecha: la igualdad.

La izquierda se benefició de un deseo generalizado de justicia social en países donde la democratización y las reformas de mercado no habían logrado reducir las profundas desigualdades. Este es el caso latinoamericano, donde líderes populistas de izquierda, con discurso antiamericano y antiglobalización, apostaron por la nacionalización y la ampliación del sector público, pero también invirtieron en programas sociales y en medidas redistributivas, de una manera más o menos clientelista. Esto da paso a la siguiente parte del marco teórico: el giro a la izquierda. 


\subsection{El giro a la izquierda}

Pink tide, turn left, new left, posneoliberalismo, entre otros, son los conceptos que múltiples académicos han acuñado para referirse a la tendencia del surgimiento de gobiernos de izquierda en los países latinoamericanos desde finales del siglo XX. La mayoría de los autores coinciden en proponer la elección de Hugo Chávez en Venezuela, en 1998, como punto de partida de esta ola de izquierda regional. Los países que conforman este giro, tomando como criterio la victoria en elecciones presidenciales, son los siguientes: Venezuela con Chavez; Chile con Lagos y Bachelet, Brasil con Lula y Rousseff; Argentina con Kirchner y Fernández; Uruguay con Mujica y Vázquez; Bolivia con Morales; Nicaragua con Ortega; Ecuador con Correa; Paraguay con Lugo; y El Salvador con Funes.

En contraste, no existe la misma cantidad de investigaciones ni consenso acerca del fin de este giro. Esto se debe a que, como se explicará con mayor profundidad más adelante, el final de esta tendencia es un proceso que aparentemente se está desarrollando desde hace pocos años. Esto quiere decir que es coyuntural y debatible. La mayoría de los trabajos referidos al giro a la izquierda latinoamericano lo entienden en contraposición, y como ruptura, con el orden político regional de los años noventa, marcado por la presencia de gobiernos que se caracterizaron por implementar reformas neoliberales en el marco del consenso de Washington.

Levitsky parte de entender el giro a la izquierda tomando en cuenta que este no implica sólo un cambio de autoridades políticas, sino también una "nueva era de experimentación de políticas en la que el gobierno expandió sus roles en la búsqueda de desarrollo, redistribución y bienestar social" (2011: p. 16). A partir de ello, sostiene que el inicio de este fenómeno es una respuesta a las consecuencias indeseadas de las políticas neoliberales. Al indagar en las causas de este fenómeno, el autor las clasifica en tres tipos: 1. Factores de largo plazo que facilitaron el surgimiento del giro a la izquierda. 2. factores históricamente contingentes que fueron detonantes del inicio del giro. 3. Condiciones internacionales que favorecieron la expansión de esta tendencia.

El primer grupo de causas incluye la desigualdad producida por las políticas neoliberales aplicadas en las décadas de 1980 y 1990. Levitsky también sitúa en este primer grupo de causas de largo plazo la institucionalización de la competencia electoral que se desarrolla una vez que se hacen menos sostenibles y tolerables los autoritarismos, los golpes militares y la exclusión arbitraria de la competencia electoral, que en la segunda mitad del ciclo habían sido utilizados para perjudicar la participación de la izquierda. En el segundo grupo de causas que fueron clave para el inicio de esta ola de izquierda, sitúa a las reformas neoliberales y a la recesión regional de 1998 a 2002. Es este segundo evento al que Levitsky le da mayor importancia como detonante en la medida en que "erosiona el apoyo público que tenían las políticas promercado" (2011: p. 18). Finalmente, en el tercer grupo de causas, que agrupa condiciones internacionales que generaron un panorama favorable para la expansión de esta tendencia, se centra en el boom de los commodities del 2002, que permitió legitimar los gobiernos de izquierda y 
evitar las posibles crisis financieras que estos pudieron haber afrontado, en caso no contaran con estas condiciones favorables.

\subsection{Unidades de análisis}

Para el presente estudio se utilizarán categorías de análisis tomadas de la literatura existente sobre el giro a la izquierda. En este sentido, dos de las tres unidades de análisis propuestas se enmarcan en el segundo tipo de causas que Levitsky llama "factores históricamente contingentes" detonantes del inicio del giro. Dentro de esta categoría se incluyen el desempeño económico y los casos de corrupción que deslegitimaron a los líderes de los gobiernos de izquierda. Si bien la mayor parte de los análisis iniciales del giro a la izquierda buscaban explicarlo a partir de factores económicos, también es importante considerar que "las identidades partidarias, los intercambios clientelistas, y la reputación de los candidatos" (Leiras 2007: 4). La tercera unidad de análisis es el papel de la derecha en la capitalización del descontento o la situación de crisis, debido a que para que esta llegue al poder, debe presentarse como una alternativa viable a los gobiernos de izquierda.

\section{REVISIÓN DE LITERATURA}

\subsection{Resultados y comportamiento electoral}

Para el caso de Argentina, se tomarán en cuenta las elecciones presidenciales (anexo 1) del 2011, en las que Cristina Fernández obtuvo una victoria aplastante, sin necesidad de segunda vuelta; y las elecciones presidenciales del 2015, en las que Mauricio Macri llega al poder. En los resultados se observa de manera clara cómo es que las preferencias electorales cambiaron de manera vertiginosa durante el tercer gobierno kirchnerista. En esa línea, las elecciones para diputados, a pesar de no ser materia central de esta investigación, respaldan la tesis que sostiene la existencia de un giro a la derecha en la Argentina: esto se aprecia en la evolución de las elecciones del 2013, 2015 y 2017 (anexo 2), en las que cambia la composición del Parlamento, pasando de una mayoría justicialista, a una mayoría de la coalición Cambiemos, de Macri.

En el caso de Brasil, se tomarán en cuenta las elecciones generales del 2010, en las que Dilma Rousseff releva a Luis Lula Da Silva en la presidencia; las elecciones generales del 2014, en las que Rousseff resultó reelegida en el cargo, pero con un porcentaje menor a su anterior victoria, tanto en la primera como en la segunda vuelta; y las generales del 2018 (anexo 3), en las que Jair Bolsonaro, representante de la derecha radical, asciende a la presidencia, acompañado de un número significativo de diputados de su partido.

La literatura sobre comportamiento electoral es bastante amplia, para este trabajo se acudirá a aquella que aborda la aprobación presidencial y sus determinantes, entre las que se encuentran las teorías del el voto económico y los efectos de la corrupción en la legitimidad del presidente. Sobre el voto económica es importante destacar que la teoría parte de la premisa de que las decisiones electorales dependen de las fluctuaciones de la 
economía. En ese sentido, los electores aplican la lógica de premio-castigo, apoyando al gobierno o candidato cuando hay prosperidad y negando su apoyo a este cuando se viven etapas de crisis (Ortiz y García, 2014: 376).

Dentro de la perspectiva del voto económico hay autores que (Powell \& Whitten, 1993; Nadeau, Niemi, \& Yoshinaka, 2002; García-Sánchez, Camargo, \& RodríguezRaga, 2011) consideran que son los factores institucionales aquellos que determinan el impacto de la economía sobre el voto de los ciudadanos: creen que es importante que los ciudadanos deben poder identificar qué tan responsable es el ejecutivo del manejo de la economía nacional; esto se puede establecer dependiendo del tiempo en el gobierno, el número de partidos y otras variables del diseño institucional. Estos estudios encontraron que, en América Latina, en aquellos países en los que el diseño institucional da mayores facultades al Ejecutivo en el manejo económico, las evaluaciones de los ciudadanos sobre el estado de la economía tienen un mayor peso sobre las decisiones electorales. Sobre el impacto de la corrupción, Zechmeister y Zizumbo (2013) sostienen que las personas que enfrentan condiciones económicas negativas aplican una penalización más alta a la aprobación presidencial por la percepción de corrupción; es decir, los ciudadanos desarrollan una mayor tolerancia a la corrupción cuando la situación económica del país es positiva.

\subsection{Actores importantes: los electores y los medios}

Respecto a los actores implicados en ambos casos, se destacan dos en especial: a los electores y a los medios de comunicación. Sobre los primeros hay que tener en consideración dos afirmaciones de Carlin, Singer y Zechmeister (2015) sobre las democracias latinoamericanas: (1) la estructura y el contenido del entorno electoral influyen en los votantes latinoamericanos y (2) las elecciones proporcionan un mecanismo por el cual los votantes eligen y sancionan a los representantes. Tomando esto en consideración, se sostiene que los ciudadanos utilizan su voto de acuerdo con la coyuntura antes que con ideales o valores: el voto está más relacionado a la situación que a la convicción. En ese sentido, ante un gobierno que parece ineficiente, probablemente los electores preferirán elegir a un candidato que suponga una ruptura con el orden anterior, aunque no necesariamente por cuestiones programáticas. Además, Carlin, Singer y Zechmeister, mencionan que el voto "es el producto final de una amplia gama de factores explicativos de la realidad social que define las orientaciones políticas, partidarias e ideológicas" (2015: 7). Esto evidencia que las decisiones que toma la ciudadanía al ir a las urnas son producto de un análisis rápido de la coyuntura actual de su país, y cómo espera que esta mejore o cambie. A partir de esto se puede comprender mejor al electorado.

Los medios de comunicación, tras el advenimiento de la tecnología digital y de las redes sociales, han jugado un papel fundamental en las elecciones. De acuerdo con un artículo publicado por la Universidad de Oregon (2017), hay seis maneras mediante las que los medios influyen en las elecciones, aquí se destacarán dos de ellas: (1) sesgos, guiones y polarización: referido a que actualmente muchos medios de comunicación 
importantes atraen audiencias partidistas, es decir, tienen como público objetivo a personas con determinadas tendencias ideológicas, que se refleja en los sesgos políticos de su cobertura. (2) Existen election-coverage scripts como las narrativas "horserace" y "game frame", enfocadas en diferenciar a los candidatos y explotar estas diferencias en favor de alguno, como sucedió con la imagen de corrupta de Hilary Clinton o la imagen de xenófoba de Donald Trump (2018: 2). En esa línea, el rol de los diarios y de los periodistas políticos también fue importante en los casos analizados, puesto que estos terminan por influir en la evaluación que realizan los votantes al momento de elegir a las próximas autoridades.

\subsection{Investigaciones sobre el giro a la derecha}

La producción académica acerca de la derecha latinoamericana en el siglo XX es escasa debido a la predominancia de gobiernos de izquierda hasta hace pocos años. Dentro de las investigaciones sobre el tema, se destacan dos autores que describen y caracterizan a estos grupos en el periodo analizado. Cannon (2016) plantea que la derecha recibió un duro golpe con el retroceso de las reformas neoliberales implantadas en los noventas. En este sentido, el autor establece que "el objetivo final de la derecha es volver a encarrilar el proyecto neoliberal, pero esta vez de una manera que será mucho más difícil de desarticular en el futuro" (Cannon 2016: 328). Además, sobre los casos analizados en este trabajo, el autor menciona que, en Brasil, la destitución de Dilma Rousseff y la presidencia de Michel Temer puede ser vista como un ejemplo de restauración de algunas políticas neoliberales, así como también la instauración de un límite para el déficit que genera el financiamiento de programas sociales (Cannon 2016: 330).

Por otro lado, Francisco López habla de una "nueva derecha" latinoamericana, que se diferencia de la derecha dictatorial de 1985 al 2000 y la derecha neoliberal de 1985 al 2000. Esta nueva derecha, que según el autor amenaza la continuidad de los gobiernos posneoliberales, tiene mecanismos como los golpes de estado "blandos", dentro de los cuales el autor sitúa la destitución de Rousseff. Otros mecanismos de la nueva derecha según el autor son las coaliciones de partidos de derecha tradicionalmente separados, dentro de los cuales coloca a Cambiemos, de Macri. Finalmente, entre las características que presenta el autor, se encuentran un discurso moderado, programas promercado menos radicales, una tendencia a desmontar los acuerdos de integración latinoamericanos, entre otros.

Finalmente, Roa (2016), plantea que, si bien la derecha ha sido tradicionalmente fuerte en América Latina, debido a sus alianzas con la Iglesia Católica, con las élites terratenientes y empresariales, las corporaciones multinacionales y los capitales extranjeros (particularmente los Estados Unidos), el ascenso y la caída de la "Nueva Izquierda" del pink tide han dejado una marca permanente en la política de la región, con implicaciones para ambos lados del espectro político. Por ejemplo, la tendencia del centrismo, particularmente fuerte entre los jóvenes latinoamericanos; esto sugiere que los líderes, partidos y movimientos de derecha que busquen ser exitosos deberán apelar a una sociedad más moderada que en el pasado. 
El hecho de que estas diversas poblaciones sean moderadas, incluida la población juvenil que creció bajo estos regímenes de izquierda, "tiene sentido cuando uno se da cuenta de que los líderes de izquierda relativamente radicales de la región (Chávez, Morales, etc.) no han intentado el tipo de transformación social extrema intentada por encarnaciones anteriores de la izquierda" (Roa, 2016: 34), como en Cuba o Nicaragua.

\section{Caso Argentina}

\subsection{Revisión histórica}

Entre 1966 y 1983, a excepción de la interrupción democrática peronista (19731979), Argentina vivió un período de dictaduras militares. Tras el breve período democrático, la dictadura militar "aplicó las políticas neoliberales del Consenso de Washington, la doctrina de seguridad nacional orientada por EU.UU., y coordinó la cruenta represión con las demás dictaduras sudamericanas mediante el Plan Cóndor" (López 2016: 48). El retorno a la democracia se produjo todavía en 1983. En ese nuevo escenario, en 1989, fue electo Carlos Menem, que, retornando a las prácticas de la dictadura, aplicó "una política económica neoliberal basada en una ola de privatizaciones de los bienes públicos, reducción de aranceles a los productos importados y desregulación de los mercados" (López 2016: 49).

Durante el gobierno menemista se aplicó la Ley de la Convertibilidad. Esta ley, decretada el 27 de marzo de 1991, fue una arriesgada política cambiaria que estableció un tipo de cambio fijo a razón de 1 dólar americano por un 1 peso convertible. Esta medida se consideró necesaria en el momento, pero tuvo terribles repercusiones para la economía argentina: "fue pensada como un chaleco de fuerza necesario para un país que en términos económicos se había vuelto loco repetidas veces, pero ese chaleco de fuerza se convirtió en sí mismo en un gran problema cuando se tornó permanente” (Rojas 2003: 108).

Debido a las consecuencias adversas de esta política, como la hiperinflación, el desempleo y desplome de la economía, se produjo una fuga masiva de capitales. Como respuesta inmediata, el gobierno de De la Rúa implementó una política de bancarización forzosa de la economía, que limitó la posibilidad de realizar retiros bancarios a nivel nacional. Esta fue la gota que derramó el vaso pues a raíz de esto se genera la insurrección del 2001. Este fenómeno implicó una profunda ruptura del orden político, ya que el levantamiento popular fue de tal magnitud que acabó con un gobierno democráticamente elegido: De la Rúa se vio obligado a renunciar. Los acontecimientos de diciembre de ese año fueron la culminación de un prolongado ciclo de luchas sociales que se gestaban desde años atrás, debido a las políticas neoliberales. Tras la renuncia de De la Rúa, hubo hasta cuatro presidentes de la República de manera provisional o interina. El ambiente era de convulsión e inestabilidad total, era "evidente la necesidad de un liderazgo político consecuente que pueda, de maneras social y moralmente aceptables" (Rojas 2003: 124).

En las elecciones del 2003 fue elegido presidente el candidato de izquierda Néstor Kirchner. Al mando del país, Kirchner “disminuyó la pobreza y el desempleo elevando el salario y creando nuevas fuentes de trabajo, lográndose un crecimiento del PIB de $8 \%$ 
(...), se duplicaron las exportaciones (...) y canceló la deuda con el FMI” (López 2016: 50). Estos logros se dieron en un contexto de boom de las exportaciones agrícolas y de subida del precio del petróleo. Sin embargo, su presidencia estuvo salpicada de varias denuncias de enriquecimiento ilícito. Acabado su gobierno asumió el mando Cristina Fernández, su esposa, quien gobernó durante dos periodos (2007-2011 y 2011-2015).

Durante estos tres periodos de kirchnerismo, el gobierno dio prioridad a políticas de inclusión social, así como medidas destinadas a atender a los sectores postergados de la sociedad durante los 90; así como también se establecieron subsidios y aranceles tributarios para favorecer a la industria local, y se retomaron las negociaciones con los sindicatos, que fueron víctimas de los embates de privatización que habían tenido los últimos gobiernos (Retamozo y Morris 2015). Como resultado de estos tres gobiernos, "la deuda en dólares de la Argentina descendió drásticamente, la pobreza se redujo en 2012 al 5.7\%, (...) la indigencia disminuyó al 1.9\%, mientras, según el BM, entre 2003 y 2009 Argentina duplicó su clase media (...) a lo que ha contribuido el sustancial incremento de las pensiones y jubilaciones” (López 2016: 51).

Sin embargo, al igual que en el gobierno de Kirchner, Fernández también se encuentra inmiscuida en escándalos de corrupción por los que está siendo procesada, así como también su hijo y funcionarios cercanos de su administración. Asimismo, los últimos años de su gestión se caracterizaron por un freno en el crecimiento económico, a la par de un incremento sustancial del déficit fiscal argentino y de la percepción de inseguridad. Respecto a lo primero, el informe de la OECD del 2017 menciona lo siguiente: “en 2015, el gasto público en Argentina fue el más alto de la región, alcanzando el 44\% del PIB. Entre 2007 y 2014, los gastos de la administración pública experimentaron el segundo mayor incremento en la región. Este aumento se puede atribuir principalmente al aumento del gasto corriente. Las presiones fiscales han aumentado en Argentina, el déficit presupuestario alcanzó el 7,4\% del PIB en 2015”. Sobre la inseguridad ciudadana, según el informe "Victimización e inseguridad subjetiva en la población urbana de la Argentina (2010-2015)", del ODSA, la inseguridad ciudadana fue la principal preocupación de los argentinos, sobre todo el 2014, cuando el $42.5 \%$ de los encuestados consideró a la inseguridad ciudadana como el principal problema público.

Durante las elecciones presidenciales del 2015, el empresario Mauricio Macri y su coalición Cambiemos, llegaron al poder. La tendencia de derecha de este político se contrapone con el legado peronista del kirchnerismo. Su victoria refleja el impacto que tuvo la inseguridad y el freno del crecimiento económico (inflación), además de los escándalos de corrupción.

\subsection{Desempeño económico}

Hasta el 2008, las cifras macroeconómicas de Argentina eran espectaculares; reflejaban el crecimiento compartido que vivía América Latina: "durante el período inicial del kirchnerismo, la Argentina tuvo una tasa anual de crecimiento de entre el $8 \%$ y 9\%" (Dabat 2013: 2). Sin embargo, estas altas tasas de crecimiento hasta el 2008 se contrastan con el modesto desempeño que tuvo la economía argentina a partir de ese año. 
En gran medida, este cambio de tendencia se debió a la crisis del 2008, a partir de la cual el crecimiento se tornó moderado.

Otro factor que generó esto fue la propia restricción externa: “a partir del 2010, el saldo del comercio exterior de la Argentina inicia un lento pero persistente declive, hasta llegar en 2015 a un déficit de U\$S 4.313 millones, el primer resultado negativo desde el 2001" (Gallo 2017: 3). Esta tendencia de caída continúa el 2011, cuando se produce un retroceso en las exportaciones, debido "al menor dinamismo de la economía mundial y por la fuerte caída de las materias primas” (Gallo 2017: 4). Según el Fondo Monetario Internacional (FMI), entre el 2011 y el 2015, el precio de los productos de exportación principales de Argentina disminuyó ostensiblemente (la soja, el trigo, el maíz y el aceite de girasol). En el aspecto fiscal, se produjo un déficit del Sector Público Nacional No Financiero (SPNNF) debido "al exceso de gastos de los gastos de capital por sobre los recursos del capital (...) en 2015, por primera vez en más de una década, los gastos corrientes exceden los ingresos corrientes, dando lugar a un déficit equivalente al 1,1\% del PBI" (Gallo 2017: 14). Entre esos gastos corrientes, el rubro más importante era el de "Prestaciones de la Seguridad Social", el cual llegó a representar un 9,2\% del PBI en el 2015; el segundo rubro más importante dentro de los gastos corrientes es el "Transferencias corrientes al sector privado".

En síntesis, el gasto público argentino se incrementó desmesuradamente a lo largo de los tres gobiernos kirchneristas al punto de sobrepasar a los ingresos corrientes, generando déficits fiscales que debían ser subsanados, o pronto se caería en recesión. Esto, sumado al fin del boom de las materias primas, llevó al estancamiento a la economía argentina. El 2015 acabó con un "déficit de comercio exterior y de cuenta corriente, y resultados negativos en el frente fiscal, tanto en lo que respecta al superávit primario total y al resultado financiero, como a la relación entre ingresos y gastos corrientes" (Gallo 2017:18).

En ese contexto, parecía urgente la aplicación de una política de ajuste fiscal. Sin embargo, el kirchnerismo, ya debilitado, no realizó esta política. Es necesario destacar que hacia el 2015, Argentina no estaba sumergida en una profunda crisis; sin embargo, era cuestión de tiempo antes de que se hagan necesarias medidas económicas de urgencia, como aquellas que se implementaron en el 2001, aquellas que desembocaron en el "argentinazo".

\subsection{Vigencia y credibilidad del liderazgo de izquierda}

La legitimidad de la izquierda en Argentina está estrechamente vinculada con la aprobación de la figura de líder que dirige al gobierno y al Partido Justicialista. Esto sucede cuando el personalismo es particularmente fuerte pues el "estilo de gobierno personalista funciona como un amortiguador de las evaluaciones negativas de la economía y un potencializador del efecto positivo del buen desempeño económico de un gobierno" (Ortiz y García, 2014: 390). Es decir, la legitimidad de Cristina Fernández, medida a través de su aprobación, es un indicador del apoyo que tiene la izquierda y su posibilidad de continuar en el poder. El segundo gobierno de Fernández estuvo plagado 
de escándalos por malos manejos y casos corrupción, que socavaron la imagen de la presidenta y de su círculo cercano, incluida su propia familia. Uno de los casos más sonados fue el de Alberto Nisman ${ }^{1}$, fiscal especial de la causa por el atentado contra la mutual judía AMIA, que hizo una fuerte denuncia a Fernández, acusándola de firmar un memorándum de entendimiento con Irán a cambio de impunidad para los sospechosos iraníes del ataque, que tuvo un saldo de 85 muertos. El caso, que tuvo lugar a inicios del 2015, tuvo un desenlace fatal para el fiscal: Nisman apareció muerto en su departamento. En medio de la conmoción social, se archivó el caso. La prensa opositora al gobierno realizaba investigaciones con la intención de demostrar algún vínculo entre el gobierno y la muerte de Nisman. A pesar de no existir una sentencia judicial, la desconfianza hacia el gobierno se incrementó considerablemente.

La primera de las causas por corrupción abiertas contra Fernández de Kirchner sucedió en 2014, tras una denuncia periodística. Esta trataba sobre la empresa Hotesur ${ }^{2}$, administradora del hotel Alto Calafate, uno de los tres que poseen los Kirchner en la provincia de Santa Cruz. Tras años de investigaciones, se procesó, en mayo del 2018, a la expresidenta y a sus dos hijos, Florencia y Máximo Kirchner, por supuesto lavado de activos y asociación ilícita. Según el expediente judicial, empresarios afines al kirchnerismo contrataron durante años habitaciones en los hoteles de los Kirchner como forma encubierta de blanquear sobornos.

Otra de las grandes acusaciones por corrupción que se ciernen sobre Fernández sucedió en el 2015, por una denuncia de la diputada Margarita Stolbizer. Esta acusó a la entonces presidente de liderar una asociación ilícita que lavó dinero proveniente de obras públicas, mediante operaciones de la inmobiliaria familiar Los Sauces ${ }^{3}$. El juzgado procesó también a sus hijos, Máximo y Florencia, y dictó un embargo sobre sus bienes por 8,4 millones de dólares. Los principales clientes de Los Sauces fueron los empresarios Lázaro Báez y Cristóbal López, quienes hicieron aportes millonarios en concepto de alquileres. El juez sospecha que fueron la fachada tras la cual se ocultó el pago de presuntos sobornos. Báez y López ahora están presos.

\subsection{Características y fuerza de la oposición}

En las elecciones del 2011, Fernández ganó en primera vuelta con más del 50\% de los votos. Esto es una demostración del grado de aprobación que llegó a tener el legado kirchnerista. Sin embargo, durante los siguientes comicios, tanto para diputados como las presidenciales, la fortaleza de los partidos de derecha, representados en coaliciones y/o bloques partidarios, fue en aumento. El candidato que finalmente logró derrotar al

\footnotetext{
${ }^{1}$ Centenera, Marc. "La muerte de Nisman, un misterio sin resolver cinco años después”, El País, 18 de enero del 2020. Disponible en: https://bit.ly/2z7AfZI

${ }^{2}$ Salinas, Lucía. "Los hoteles y la inmobiliaria de los Kirchner seguirán intervenidas hasta que inicie el juicio", Clarín, 24 de febrero del 2020. Disponible en: https://www.clarin.com/politica/hotelesinmobiliaria-kirchner-seguiran-intervenidas-inicie-juicio_0_Zq4DIcHy.html

3 "Los Sauces: Margarita Stolbizer pide a la Justicia que Cristina Kirchner vaya a juicio oral", Clarín, 6 de julio del 2018. Disponible en: https://www.clarin.com/politica/sauces-margarita-stolbizer-pide-justiciacristina-kirchner-vaya-juicio-oral_0_SJ98Qb6fQ.html
} 
kirchnerismo en las presidenciales fue Mauricio Macri, un exitoso empresario que había sido jefe de Gobierno de Buenos Aires en dos ocasiones consecutivas y que es fundador de la coalición Cambiemos, integrada por partidos como Unión Cívica Radical, históricamente de derecha. Esto quiere decir que los partidos de derecha, a pesar de no obtener triunfos electorales significativos, se mantuvieron relativamente activos durante el kirchnerismo. Lo suficientemente activos como para capitalizar el momento de debilidad del kirchnerismo, desgastado por la desaceleración económica y los casos de corrupción.

Los resultados electorales de las presidenciales del 2015 reflejan que el voto de la población estuvo fuertemente influenciado por los errores que habían cometido Fernández y sus allegados políticos. Esto llevó a que, si bien el candidato oficialista Daniel Scioli resultó como el más votado en primera vuelta, perdió la presidencia en la segunda vuelta por un estrecho margen, ante un candidato de derecha como Macri. La fuerza de la oposición también puede ser medida respecto a la composición de los resultados de los comicios parlamentarios del 2017, en los que Cambiemos aumentó su número de representantes en la Cámara: obtuvieron el $41.75 \%$ de los votos. Estos resultados parecen comprobar un giro político en las preferencias electorales de los argentinos.

\section{Caso Brasil}

\subsection{Revisión histórica}

Durante la campaña electoral presidencial de 2002, Lula representó una propuesta en contraposición a las políticas neoliberales de Cardoso, su antecesor (Hunter 2011: 307 en Levitsky 2011). La situación de freno económico y desempleo que acompañó el último año del gobierno de su antecesor es clave para explicar popularidad del discurso de izquierda del entonces candidato por el Partido de los Trabajadores. La gestión presidencial de Lula, sin embargo, no fue del todo consecuente con su postura como candidato, ya que implementó reformas y políticas más moderadas de lo esperado. Sin embargo, estas políticas orientadas más bien al funcionamiento y el crecimiento de la economía fueron clave en el desarrollo económico que experimentó Brasil y en el posicionamiento que logró en la política internacional.

A pesar de esta orientación de su gestión, el discurso y la imagen de Lula seguía tendiendo hacia la izquierda. Para el 2008, durante su segunda gestión, Brasil había alcanzado una importante tasa de crecimiento económico, la capacidad de consumo de la población había aumentado y el desempleo había disminuido. Sin embargo, esta situación no fue producto exclusivamente del desempeño de la gestión de Lula, sino que influyeron también factores internacionales favorables como el despegue de nuevas potencias como China e India (Kingstone en Weyland, 2010: 106). A pesar de que Lula no implementó reformas sustanciales respecto a las políticas sociales consecuentes con su discurso, la reducción del desempleo y la disminución de la pobreza que generó el crecimiento económico logró asegurar su popularidad. 
Tras el segundo gobierno de Lula, fue electa su sucesora del PT, Dilma Rousseff. Si bien Rousseff, inicialmente, continuó con las políticas del gobierno de Lula, su gobierno tuvo que enfrentar un escenario de crisis económica y política. La disminución considerable del PBI y las protestas ciudadanas que denunciaban la insuficiencia de los servicios sociales, uno de los legados del gobierno de Lula (Barros en Weyland 2010: 60), iniciaron el debilitamiento de su mandato. En el 2014, tras ser reelecta Rousseff, Brasil entra su peor contracción económica desde 1930 (Van Dyck 2016: 9), que pronto fue acompañada por una crisis política generada por los escándalos de corrupción que involucraban a altos funcionarios del gobierno, incluida la mandataria. Esta crisis se extendió hasta el 2016, culminando con la destitución de su cargo. A continuación, se desarrolla a profundidad este último periodo de aparente crisis del gobierno de Dilma Rousseff como indicador de crisis del periodo de gobiernos de izquierda en Brasil.

\subsection{Desempeño económico}

El desempeño económico de un gobierno es fundamental para determinar la capacidad del Estado para brindar bienes y servicios públicos a la población. En este sentido, es preciso considerar este factor como fundamental para entender la crisis de un régimen político y, en este caso, de un modelo de Estado. Los gobiernos de Lula da Silva y el primero de Rousseff se caracterizaron por la expansión del estado, con una orientación hacia políticas de redistribución y de bienestar social (Levitsky 2011: p. 16). Estas políticas requirieron un incremento del gasto público, pero fueron posibles gracias al panorama económico positivo. Sin embargo, esta orientación enfrentó severos problemas durante el segundo gobierno de Rousseff, debido a la recesión de la economía brasileña. Grugel y Riggirozzi (2018) sostiene que desde antes de la reelección de Rousseff ya se advertía una desaceleración en el crecimiento económico del país; sin embargo, por encontrarse en su fase inicial, esta aún no era relacionada con la gestión del gobierno. Esta tendencia, sin embargo, continuó manifestándose de manera drástica sumiendo la economía en una recesión de magnitudes históricas, tal como puede interpretarse en la evolución del PBI observable en el siguiente gráfico del Instituto Brasileño de Geografía y Estadística (IBGE).

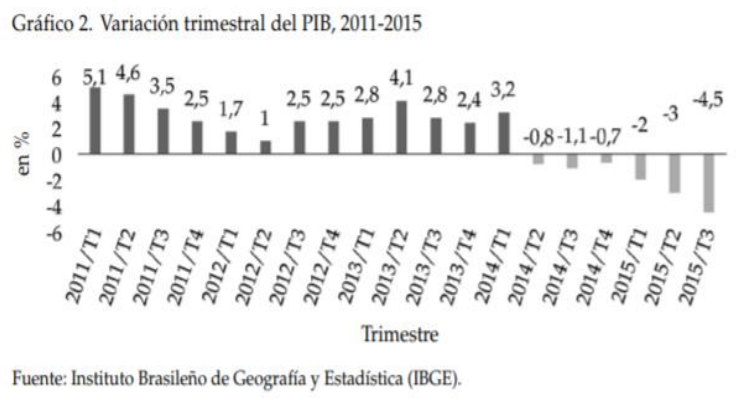

La recesión se intensificó durante el primer año del segundo mandato de Rousseff. El gobierno atribuyó esto, en un primer momento, a factores internacionales. Sin embargo, tal como proponen Serrano y Summa, "un análisis de la data de la economía 
brasileña revela que la política macroeconómica interna, tanto monetaria como fiscal, fue en gran parte responsable de la fuerte desaceleración en el crecimiento del producto de Brasil.” (Serrano y Summa 2013: 18). Grugel y Riggirozzi (2018) coincide en argumentar que la imprecisión y la demora de los ajustes macroeconómicos de la gestión de Rousseff, tomando en cuenta el panorama económico, fue una de las causas que potenciaron la inestabilidad. El autor enfatiza en el aumento de gasto público del gobierno cuando ya existía una reducción de la recaudación fiscal, lo cual hizo explotar el déficit en el 2015 (Grugel y Riggirozzi 2018). Las medidas que tuvo que tomar Rousseff ante esta situación crítica fueron de austeridad. En este sentido, "se convirtieron en imperativos recortar los gastos sociales, reducir el crédito de los bancos públicos, subastar propiedades del Estado y aumentar tasas para llevar el presupuesto de vuelta a una situación de superávit primario" (Anderson, 2016: 3). La combinación del decrecimiento económico y las medidas de ajuste implementadas de urgencia disminuyeron considerablemente la calidad de vida de la población. Esto se evidencia en el aumento de la tasa de desempleo tal como puede observarse en el siguiente gráfico, elaborado por del Instituto Brasileño de Geografía y Estadística (IBGE)

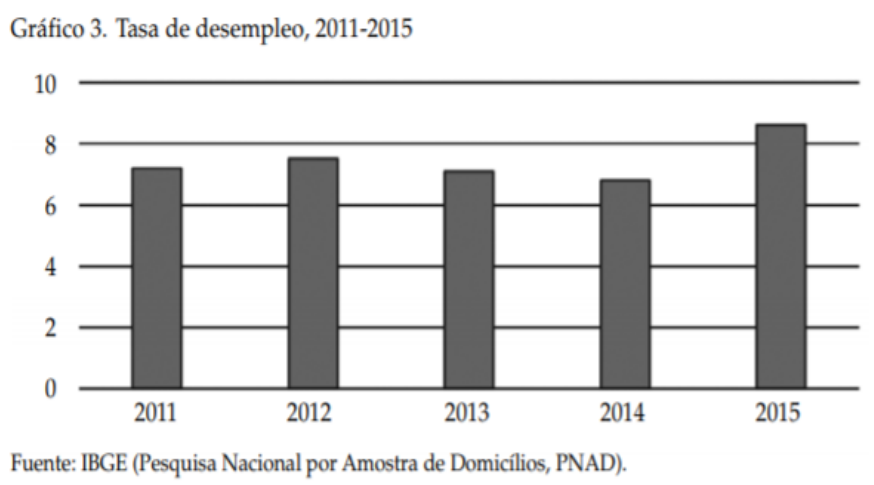

\subsection{Vigencia y credibilidad de la izquierda}

Otras situaciones críticas que acompañaron la segunda gestión de Dilma desde el primer mes de su mandato fueron los destapes de casos de corrupción y las movilizaciones sociales contra el gobierno. De la misma manera muchos otros políticos especialmente relacionados con el partido se verían implicados en estos escándalos, e incluso Lula, líder histórico de la izquierda y el PT, serían juzgado por casos de corrupción. Esta situación sería otro de los factores que habrían determinado la erosión de la legitimidad y la credibilidad de las gestiones y los líderes del giro a la izquierda en el caso de Brasil. Esta situación culminó con la destitución de la mandataria y el fin del periodo dominado por el PT en el 2016. A continuación, se hará un recuento de los golpes que recibió la legitimidad y aprobación de Rousseff y el Partido de los Trabajadores.

La operación Lava Jato reveló, en 2014, que desde años atrás se había gestado una red de corrupción entre políticos, empresas estatales y contratistas. Para Doval y Actis, fue especialmente simbólico el hecho de que esté involucrada la empresa Petrobras que, tal como proponen, era considerada el "corazón" del gobierno del PT debido a que era la principal fuente para sostener sus políticas, pues aportaba el 10\% del PBI (Doval et. al 
2016: 123). Durante esta situación, que tuvo efecto en una disminución drástica en la aprobación de la mandataria en su primer año del segundo mandato: según las encuestas realizadas por el Ibope (2015), Rousseff tuvo sólo el 9\% de aprobación presidencial en diciembre del 2015, resultado similar al de meses anteriores. En medio de esta caída en picada de la aprobación presidencial, empiezan dos procesos que Nunes y Ranulfo (2017) consideran como los principales responsables del impeachment en el 2016.

En primer lugar, surgen manifestaciones en contra del gobierno que demuestran el rechazo popular. Sin embargo, los autores mencionan que este proceso no se sostuvo en el tiempo y las manifestaciones perdieron apoyo. El segundo proceso, que se refiere a las acciones tomadas por los partidos en las cámaras legislativas y cuenta con mayor peso en la explicación de los autores, será tratado en la sección de características y fuerza de la oposición. Esta situación de deslegitimación del gobierno de Rousseff -que culminó con su destitución y el ascenso de Michel Temer a la presidencia-, sin embargo, no erosionó completamente la legitimidad del Partido de los Trabajadores. Evidencia de esto es el apoyo que tuvo en la campaña para las elecciones del 2018 Lula da Silva. Esto cambió drásticamente con la sentencia contra el candidato y líder histórico del PT por corrupción y lavado de dinero, lo cual implicó su separación de la contienda electoral. Su sucesor como candidato del PT, Haddad, no logró alcanzar la popularidad esperada en las últimas semanas de campaña, lo que se presta a la interpretación de que ese último respaldo a la candidatura, representante de los gobiernos del giro a la izquierda brasileño, era más a la persona de Lula que a lo que el PT representaba.

\subsection{Características y fuerza de la oposición}

Para analizar las características de la oposición y su desenvolvimiento en la situación de crisis del largo periodo de gobierno del PT, es necesario tener en cuenta dos momentos. El primero de estos es el proceso de impeachment y la gestión posterior al mando de Temer, en la cual la reconfiguración de los partidos de las cámaras legislativas jugó un papel importante. En segundo lugar, es preciso analizar la campaña de las elecciones del 2018, en la cual surge una opción de oposición con un representante que hubiera sido poco creíble años antes: Jair Bolsonaro. El discurso cargado de populismo y presentado como antítesis a la tendencia de los gobiernos del PT, logró aprovechar el descontento y la impopularidad del escenario político previo.

La situación tensa que existía entre el poder ejecutivo y el legislativo se hizo aún más problemática en este periodo de crisis e inestabilidad. Esto se manifestó principalmente en el conflicto que hubo entre la designación de gabinete ministerial de Rousseff y la aprobación de este por parte del Movimiento Democrático Brasileño (MDB). En este contexto, el legislador Cunha, miembro del partido en mención, inició una campaña dentro del legislativo en contra del ejecutivo, teniendo como bandera el proceso de impeachment contra la entonces mandataria (Nunes et. al 2017: 284). Finalmente, a partir de acusaciones de alteración de información económica en beneficio de su gestión, la destitución de Rousseff fue votada y aprobada en el ejecutivo. 
En segundo lugar, cabe analizar el desempeño y el cambio de las características de la oposición en la contienda electoral del 2018. Durante este proceso, fue sorprendente el crecimiento inesperado de la popularidad de Jair Bolsonaro: postuló con el Partido Social Liberal, partido de derecha con menor relevancia en los procesos electorales previos. Bolsonaro, con un discurso populista y personalista terminó por circunscribir el partido a su figura. La plausibilidad de la propuesta del candidato ultraderechista es producto de la capitalización de la situación de insatisfacción con el establishment que tenía el pueblo brasileño. Esta situación fue producida por la coincidencia de la crisis económica, política, y el aumento de la violencia en las calles de Brasil.

\section{COMPARACIÓN Y CONTRASTACIÓN DE CASOS}

Como se explicó anteriormente, el desempeño económico es un determinante de la aprobación o desaprobación de un gobierno. En caso este no sea el esperado por la población, las evaluaciones que realicen a quienes se encuentren en los cargos públicos serán negativas. En los casos brasileño y argentino se puede observar que el desempeño económico que tuvieron en los últimos años sus respectivos gobiernos de izquierda contrasta con las cifras extraordinarias de crecimiento suscitadas hasta el 2010. En el caso de Argentina, no se produjo una recesión como tal durante el segundo gobierno de Cristina Fernández; sin embargo, el crecimiento se había estancado y la economía había perdido la solidez que los commodities le habían garantizado por tanto tiempo. La combinación entre el incremento del gasto fiscal por la inversión en más políticas de desarrollo e inclusión social y los factores internacionales desfavorables, reflejados en la disminución de las exportaciones, tuvieron un desenlace negativo en el balance económico argentino hacia fines del 2014 y contribuyó a un desalentador desempeño en el 2015, año en el que Mauricio Macri fue elegido presidente. Es menester remarcar que este desempeño económico negativo fue expuesto y explotado por los medios de comunicación de opositores. En el caso de Brasil sí se dio una recesión extraordinaria desde el 2014, que no se debió tanto a factores internacionales como a las deficientes políticas macroeconómicas del Estado. En este caso, las medidas de ajuste que el gobierno tuvo que aplicar para contrarrestar la crisis implicaron la reducción de presupuesto de los programas sociales, del crédito de los bancos públicos y el aumento de la tasa de interés. Esta situación, sumada a la alta y creciente tasa de desempleo, desencadenó el descontento del pueblo y fue motivo de múltiples movilizaciones en contra del gobierno y sus políticas.

La reputación de un político es aquello que le permite tener legitimidad para tomar decisiones y, sobre todo, para aspirar a mantenerse en el cargo luego de terminado un período determinado; incluso si el político no pueda postular nuevamente, le da posibilidades a su partido en los siguientes comicios. La credibilidad de las lideresas de Argentina y Brasil se vincula con el bienestar de la población, debido a que sus gobiernos se han cimentado sobre el apoyo de los sectores populares y desfavorecidos.

En el caso argentino hubo varios escándalos por corrupción e incluso un presunto asesinato, que involucran a Cristina Fernández, a miembros de confianza de su 
administración, miembros de su partido e incluso a su propia familia. Sin embargo, los procesos judiciales contra ella recién cobraron fuerza luego de que haya dejado el poder, pues mientras fue presidenta tuvo mayor control político sobre estas situaciones. No obstante, la prensa argentina se encargó de difundir estas noticias y de condenar los actos de Fernández de Kirchner, así como también de visibilizar el posible encubrimiento y las influencias de la presidenta en el Poder Judicial, direccionando a la opinión pública contra la administración Fernández. Esto, sumado a que la gestión del gobierno tenía una labor menos eficiente que en las dos gestiones anteriores, sobre todo en la economía y la seguridad ciudadana, contribuyó a que la opinión pública cambiase su perspectiva sobre Fernández.

En el caso de Brasil, la legitimidad de Rousseff se vio disminuida debido a casos de corrupción ligados a funcionarios del gobierno (Petrobras) y a la acusación por alterar las cifras de las cuentas públicas. El primer caso llevó a grandes movilizaciones en contra el gobierno, mientras la segunda acusación, dirigida específicamente a la mandataria, fue el fundamento del proceso de su destitución. El papel del Poder Judicial y de los parlamentarios fue crucial para que ambos procesos tomen relevancia. Estos procesos, sin embargo, no debilitaron del todo la legitimidad del partido de los trabajadores y su líder histórico: Lula da Silva. Esto se evidenció en la popularidad que tuvo en campaña para las elecciones del 2018. Hasta ese momento aparentemente la deslegitimación había afectado principalmente a Rousseff. Sin embargo, cuando Lula fue condenado por corrupción, parece haberse abierto el camino para Bolsonaro.

Los factores analizados hasta el momento generaron en ambos casos el descontento de la población o la percepción de caducidad del modelo de gobierno de las gestiones previas. Las consecuencias de ello en la evolución de los resultados electorales dependieron sustancialmente de la capitalización de la situación de crisis por parte de la oposición o los partidos de derecha. En este sentido, es importante diferenciar las características que tenían estos en cada caso, enfatizando en el nivel de institucionalización y de estructura partidaria. En Argentina, Macri llegó al poder de la mano de la coalición Cambiemos, conformada por partidos políticos tradicionales de derecha, como Unión Cívica Radical (fundado en 1891) y el Partido Conservador Popular (fundado en 1958). Por su lado, Mauricio Macri ya contaba con un perfil y antecedentes políticos que lo acreditaban como líder y cara visible de la coalición: fue jefe de gobierno por Buenos Aires en dos períodos consecutivos (2007-2011 y 2011-2015). Es importante recordar que varios de los partidos y movimientos que se adhirieron a esta coalición en el 2015, habían competido comisiones electorales previamente, sin mayor éxito.

En el caso de Brasil, Jair Bolsonaro, exmilitar sin experiencia política previa, tuvo un ascenso inesperado. Este candidato postuló a las elecciones del 2018 con un partido que había tenido resultados irrelevantes en los procesos electorales previos (PSL), llegando a no conseguir un solo curul en el Parlamento. Pareciera que el discurso discurso populista y radical de Bolsonaro modificó drásticamente la trayectoria electoral del partido. En este sentido, puede afirmarse que, más que un trabajo de largo plazo e intencional para sacar provecho de la crisis del establishment, el ascenso de Bolsonaro se 
debió en mayor proporción a factores coyunturales, pues la derecha no tenía partidos con la articulación que existía en Argentina.

\section{Conclusiones}

En conclusión, la hipótesis fue acertada, pues los tres factores planteados inicialmente tuvieron los efectos esperados en ambos casos. Sin embargo, el nivel explicativo de cada factor fue variando. Por ejemplo, la derecha argentina llegó al poder a través de una coalición conformada por partidos institucionalizados y con un candidato con experiencia en de gobierno. Mientras que en Brasil el partido del que salió el nuevo presidente era uno sin mayor protagonismo desde su fundación, relativamente reciente. Por su lado, Bolsonaro no tenía experiencia de gestión anterior, sino que emergió como un underdog, favorecido por la coyuntura. Esto quiere decir que la fuerza de la oposición, medida a partir de la viabilidad de la propuesta de derecha, en contraposición con los gobiernos de izquierda, es significativamente distinta.

Sobre el desempeño económico, si bien ambos países afrontaron dificultades por la caída de los commodities, la reducción de exportaciones y el aumento desmesurado del gasto fiscal, Brasil se vio afectado por esta situación en mayor medida que Argentina. No obstante, al ser resultados económicos inferiores a los obtenidos anteriormente, por ambos gobiernos, terminó por impulsar a los votantes a optar por opciones opuestas.

Respecto a la credibilidad de las líderes del gobierno, en ambos casos se apreciaron situaciones que vinculaban directa e indirectamente a la Rousseff y Fernández, así como a sus funcionarios e incluso familiares, en escándalos de corrupción. Estos fueron utilizados por la oposición y los medios de comunicación contrarios al gobierno para socavar la imagen de la presidencia.

Los candidatos de derecha, en las elecciones del 2015 en Argentina y del 2018 en Brasil, sacaron provecho del descontento y desaprobación a la gestión anterior para marcar diferencias y presentarse como propuestas no sólo distintas en lo ideológico, sino también en lo práctico: se presentaron como la solución a los problemas ocasionados por los gobiernos de izquierda.

A partir del recuento realizado, se puede afirmar que, a diferencia del previo giro a la izquierda en la región, el aparente contragiro a la derecha no necesariamente logrará expandirse con la misma magnitud en las democracias latinoamericanas. Se tiene como ejemplo más cercano de esto la victoria en del candidato presidencial justicialista Alberto Fernández en Argentina. Esto se debe a que los factores que posibilitaron la victoria de la derecha en los dos casos analizados no son todos de carácter regional, como sí lo fueron en el pink tide. A inicios del siglo XXI, este giro surgió como una respuesta a las reformas neoliberales que se dieron de manera generalizada en la región, por lo que existía un escenario económico común. Sin embargo, las condiciones que han posibilitado en Argentina y Brasil el ascenso de la derecha no son homogéneos en la región. Además, ya no existe un modelo común al cual oponerse, como sí sucedió con el modelo neoliberal: los gobiernos de izquierda ante los que surgen este auge de la derecha han tenido distintos 
niveles de desempeño y distintos tipos de políticas, así como también diferente arraigo. Por otro lado, en los dos casos, los gobiernos del giro a la izquierda tuvieron inicialmente un buen desempeño económico, que luego fue drásticamente cambiado por el estancamiento progresivo. Este nivel de bonanza económica es una característica con la que no contó el gobierno de Macri, ni el de Bolsonaro actualmente.

Además, los casos de corrupción propuestos como detonantes claves en este supuesto giro a la derecha de ambos países han alcanzado a las presidentes y otros funcionarios de alto rango, algo que no ha sucedido en la mayoría de los países de la región; esto hace difícil que este modo de deslegitimación pueda ser capitalizado por otros movimientos de derecha.

Finalmente, a pesar de que los factores se plantearon como las variables que determinan un posible giro a la derecha fueron acertados, la conclusión a la que se llega no es la que esperada. La derecha que gobernó en Argentina es diferente a la derecha que gobierna en Brasil. En primera instancia, porque una fue elegida a través de una coalición conformada por partidos tradicionales de derecha y la otra por un partido que había perdido relevancia a través del tiempo, tanto en la discusión política como en las elecciones. Macri representa a una derecha liberal, en contraste con Bolsonaro, que es parcialmente liberal en lo económico y extremo-conservador en lo político.

Asimismo, la derecha argentina contaba con un mayor respaldo, pues los partidos tradicionales de derecha se habían mantenido activos, a pesar de su perfil bajo. Si bien Bolsonaro, e incluso su hijo, tuvo contundentes victorias electorales, no es probable augurar un futuro gobierno donde el presidente tenga gobernabilidad y legitimidad para realizar las políticas de reforma económica, por ejemplo, pues el PT aún tiene una presencia importante en el Parlamento. Esto nos lleva a ser cautelosos con el alcance regional que podría tener este aparente giro político, pues, a pesar de que, en principio, se han reunido condiciones similares a las que se tuvieron en el giro a la izquierda, podría tratarse también de un recambio político por desgaste del gobierno. En corto, este supuesto giro a la derecha no representa necesariamente una derechización de la sociedad, es decir, los ciudadanos no necesariamente se identifican con la derecha. En estos dos casos parece que se está ante un profundo rechazo por casos de corrupción, antes que una derechización de la sociedad.

Pero más allá de eso, las tendencias electorales reflejan un cambio en las preferencias del electorado brasileño y argentino, de discursos populistas de desarrollo socioeconómico hacia discursos populistas de crecimiento económico. No es posible demostrar que la victoria de Duque, en Colombia, ni la de Piñera en Chile formen parte de este giro a la derecha, pero, de serlo, podría tratarse de una nueva era en la política latinoamericana, una que también se ve reflejada en Occidente (Francia, Austria y República Checa). Al parecer sólo el tiempo podrá determinar si esta evolución en las elecciones argentinas y brasileñas implicarán un cambio de paradigma; el revés en la Argentina y el debilitamiento de Bolsonaro parecen indicar lo contrario. 


\section{REFERENCIAS}

Actis, E. and Creus, N. (2018). "América Latina en la nueva bipolaridad emergente". Revista Nueva Sociedad, Outubro.

Bowen, James (2011). "The Right in "New Left" Latin America". Journal of Politics in Latin America. 2011, Vol. 3 Issue 1, p99-124

Caparrós, Martín (2016) “_Fracasó la izquierda latinoamericana?”. The New York Times. 03/01/20. https://www.nytimes.com/es/2016/09/16/fracaso-la-izquierdalatinoamericana/

Cameron, M. A., \& Hershberg, E. (2010). “Latin America's left turns: Politics, policies, and trajectories of change". Boulder: Lynne Rienner Publishers.

Centera, M. y Rivas, F. (2018). "Las causas judiciales contra Cristina Kirchner". El País. Buenos Aires, 19/08/18. Consulta: 10/01/19. https://elpais.com/internacional/2018/08/17/argentina/1534524746_729644.html

Cannon, B. (2016). Inside the Mind of Latin America's New Right. NACLA Report on the Americas, 48(4), 328-333. https://doiorg.ezproxybib.pucp.edu.pe/10.1080/10714839.2016.1258275

Carlin, Ryan, Matthew Singer y Elizabeth Zechmeister (2015). The Latin American Voter: Pursuing Representation and Accountability in Challenging Contexts. Ann Arbor: University of Michigan Press.

Dabat, Alejandro (2013). "El rumbo de la economía argentina bajo el kirchnerismo". Economíaunam. Ciudad de México, 2013, volumen 9, número 26, pp. 43-67. Consulta:

$07 / 01 / 20$. http://www.economia.unam.mx/publicaciones/nueva/econunam/26/02dabat.pdf

Dominguez, Jorge (2016). “¿Qué ha pasado con la izquierda en Latinoamérica?”. Foreign Affairs Latinoamérica. Volumen 16 Número 3.

Doval, G. y Actis, E. (2016). The Political and Economic Instability of Dilma Rousseff's Second Government in Brazil: Between Impeachment and the Pragmatic Turn. India Quarterly: A Journal of International Affairs, 72(2), 120-131.

Grugel, J. y Riggirozzi, P. (2018). "Neoliberal disruption and neoliberalism's afterlife in Latin America: What is left of post-neoliberalism?". Critical Social Policy. Vol. 38

Gallo, Marcos (2017). La economía argentina durante el período 2004-2017: ciclo expansivo, restricción externa y retorno de la valorización financiera. Recuperado el 03/01/20 de: http://nulan.mdp.edu.ar/2757/1/gallo-2017.pdf

García-Sánchez, Miguel; Camargo, Gabriel N. y Rodríguez-Raga, Juan (2010). Institutions, Accountability, and Conditional Economic Voting in Latin America A Hierarchical Analysis.

Gerchunoff, Pablo y Osvaldo Kacef (2016). “¿Y ahora qué hacemos? La economía política del kirchnerismo”. Análisis. Buenos Aires, 2016, número 6, pp. 4-35. Consulta: 03/01/20 https://library.fes.de/pdf-files/bueros/argentinien/12674.pdf

Hofman, Andre (2017). "Crecimiento económico y productividad en Latinoamérica. El proyecto LA-KLEMS”. Trimeste Económico. Vol. 84 Issue 334, p259-306

Hunt, Sarah (2016). "Rethinking the Politics of the Shift Left in Latin America: Towards a Relational Approach”. Bulletin of Latin American Research. Vol. 35 Issue 4, p437-451.

Levitsky, S., \& Roberts, K. M. (2011). "The resurgence of the Latin American left". Baltimore: Johns Hopkins University Press.

Lopez, Francisco (2016). “América Latina: crisis del posneoliberalismo y ascenso de la nueva derecha". Buenos Aires: CLACSO. 
Leiras, M. (2007). Latin America's Electoral Turn: Left, Right, and Wrong. Constellations: An International Journal of Critical \& Democratic Theory, 14(3), 398-408. https://doi-org.ezproxybib.pucp.edu.pe/10.1111/j.14678675.2007.00454.x

Moreira, Carlos y Sebastián Barbosa (2010).

"El kirchnerismo en Argentina: origen, apogeo y crisis, su construcción de poder y forma de gobernar". Revista Sociedad de Cultura. Goiania, vol. 13, núm. 2, pp. 193-200. Consulta: 07/01/20. http://www.redalyc.org/pdf/703/70316920005.pdf

Navas, Alejandro (2014). Izquierda y Derecha: ¿una tipología válida para un mundo globalizado? Revista de Comunicación, 13, 163-176

Nadeau, Richard; Niemi, Richard. G., y Yoshinaka, Antoine (2002). “A Cross-National Analysis of Economic Voting: Taking Account of the Political Context Across Time and Nations". Electoral Studies 21 (3): 403-423.

Nunes y Ranulfo (2017). Impeachment, Political Crisis and Democracy in Brazil. Revista de Ciencia Política, vol. 37, $\mathrm{N}^{\circ}$ 2, p. 281-304.

Ortiz Ayala, Alejandra y Miguel García Sánchez (2014). "Porque te quiero te apoyo. Estilo de Gobierno y Aprobación Presidencial en América Latina." Revista de Ciencia Política, Vol. 34, No. 2, pp. 373-398.

Powell Jr, G. Bingham, y Whitten, Guy (1993). “A Cross-National Analysis of Economic Voting: Taking Account of the Political Context". American Journal of Political Science 37 (2): 391-414.

Romero, Simon (2016). “¿La era de la izquierda se desvanece en América Latina?”. The New York Times. 5/01/20 https://www.nytimes.com/es/2016/10/05/la-era-de-laizquierda-se-desvanece-en-america-latina/

Rowland, Aron (2015). "The State in Context: Latin America's New Left and the Legacies of the State". Current Perspectives in Social Theory. 2015, Vol. 34, p125-153

Seligson, Mitchell (2002). "The impact of corruption on regime legitimacy: A comparative study of four Latin American countries." Journal of Politics, Num. 64, pp. 408-433.

Seawright, Jason (2013). "Voter Affect and the Demise of Party Sytems." Capítulo 6 en Party System Collapse. California: Stanford University Press.

Torrico, Mario (2017). “¿Fin del giro a la izquierda en América Latina?: Gobiernos y políticas públicas". México: FLACSO.

University of Oregon (2017). Six ways the media influence elections. Consulta: 10/01/20. https://journalism.uoregon.edu/news/six-ways-media-influences-elections

Van Dyck, Brando (2016). “QQué tan grave es la crisis brasileña?”. Foreign Affairs Latinoamérica. Volumen 16 Número 3.

Weyland, K. G., Madrid, R. L., \& Hunter, W. (2010). "Leftist governments in Latin America: Successes and shortcomings". Cambridge: Cambridge University Press

Wainer, A. (2018). "Economía y política en la Argentina kirchnerista (2003-2015)" Revista Mexicana de Sociología. Ciudad de México, número 2, pp. 323-351. Consulta: 07/01/20 http://www.scielo.org.mx/pdf/rms/v80n2/0188-2503-rms-8002-323.pdf

Zechmeister, Elizabeth J. y Daniel Zizumbo-Colunga (2013). “The Varying Political Toll of Concerns about Corruption in Good versus Bad Times" Comparative Political Studies, Vol. 46, Nro. 10, pp. 1190-1218. 
Politai: Revista de Ciencia Política, 2019, Año 10, segundo semestre, № 19, pp. 109-135

\section{AneXos}

\section{Anexo 1}

\section{Total País}

Presidente y Vicepresidente - Generales - 23 de octubre de 2011

La fórmula Fernández de Kirchner - Boudou,

La fórmula Fernández de Kirchner - Boudou,
obtiene el triunfo de las elecciones con el $54,11 \%$

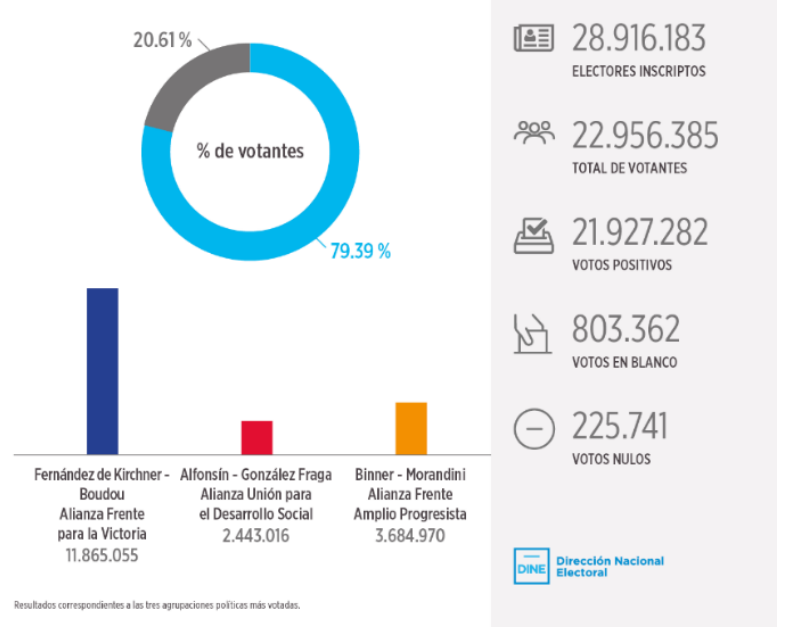

Resultados de las elecciones generales de presidente y vicepresidente (primera vuelta del 2011) - Dirección Nacional Electoral

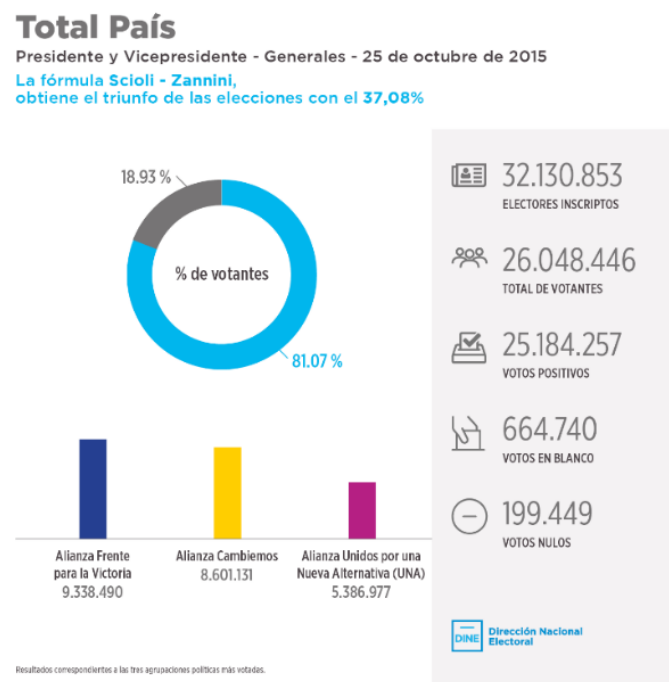

Resultados de las elecciones generales de presidente y vicepresidente (primera vuelta del 2015) - Dirección Nacional Elector 


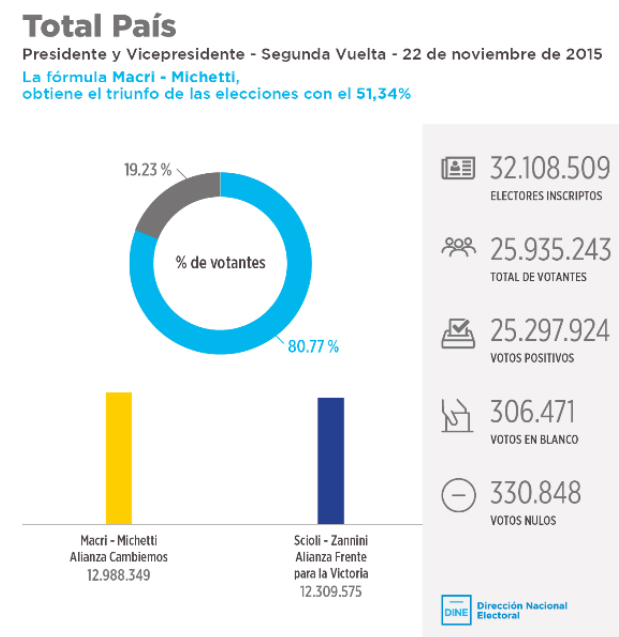

Resultados de las elecciones generales de presidente y vicepresidente (segunda vuelta del 2015) - Dirección Nacional Electoral



Composición de la Cámara de Diputados del 2013 - Dirección Nacional Electoral (Wikipedia)

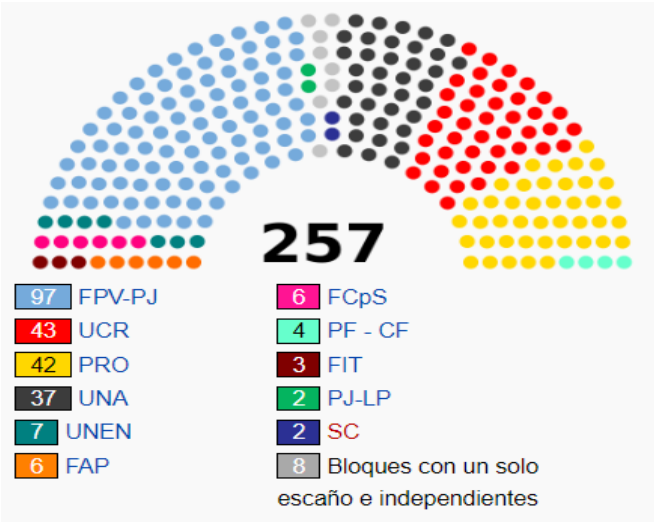

Composición de la Cámara de Diputados del 2015 - Dirección Nacional Electoral (Wikipedia) 


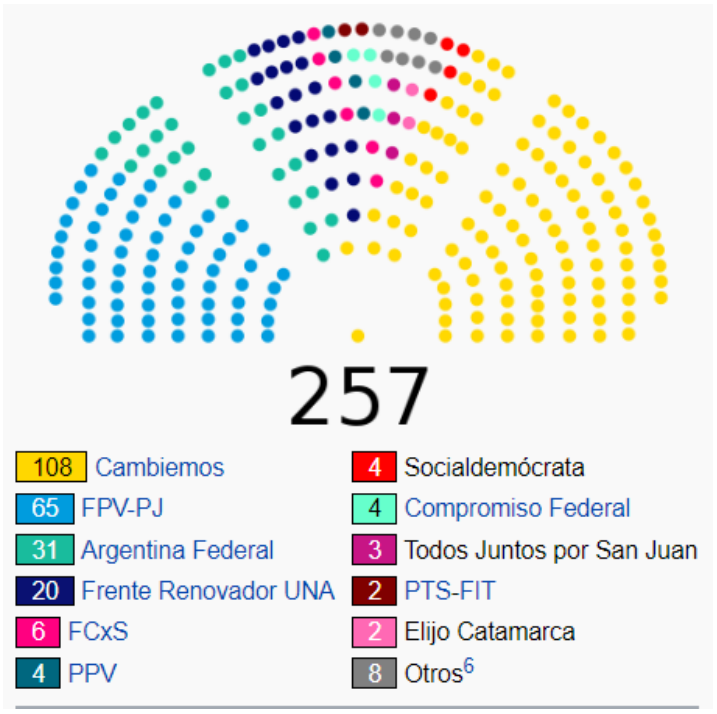

Composición de la Cámara de Diputados del 2017 - Dirección Nacional Electoral (Wikipedia)

Anexo 3

\begin{tabular}{|c|c|c|c|c|c|c|c|c|}
\hline UF $\uparrow \equiv$ & Cargo & Candidato & Idade & $\mathrm{Nr}$ & Partido & $\begin{array}{l}\text { Votação } \\
\text { Nominal }\end{array}$ & Situação & Sexo \\
\hline $\mathrm{BR}$ & PRESIDENTE & IVAN MARTINS PINHEIRO & 64 & 21 & РСB & 39.136 & $\begin{array}{l}\text { NÃO } \\
\text { ELEITO }\end{array}$ & MASCULINO \\
\hline $\mathrm{BR}$ & PRESIDENTE & RUI COSTA PIMENTA & 53 & 29 & PCO & 12.206 & $\begin{array}{l}\text { NÃO } \\
\text { ELEITO }\end{array}$ & MASCULINO \\
\hline $\mathrm{BR}$ & PRESIDENTE & JOSE LEVY FIDELIX DA CRUZ & 59 & 28 & PRTB & 57.960 & $\begin{array}{l}\text { NÃO } \\
\text { ELEITO }\end{array}$ & MASCULINO \\
\hline BR & PRESIDENTE & JOSÉ SERRA & 68 & 45 & PSDB & 33.132 .283 & $\begin{array}{l}2^{\circ} \\
\text { TURNO }\end{array}$ & MASCULINO \\
\hline BR & PRESIDENTE & JOSE MARIA EYMAEL & 71 & 27 & PSDC & 89.350 & $\begin{array}{l}\text { NÃO } \\
\text { ELEITO }\end{array}$ & MASCULINO \\
\hline $\mathrm{BR}$ & PRESIDENTE & PLÍNIO SOARES DE ARRUDA SAMPAIO & 80 & 50 & PSOL & 886.816 & $\begin{array}{l}\text { NÃO } \\
\text { ELEITO }\end{array}$ & MASCULINO \\
\hline BR & PRESIDENTE & JOSÉ MARIA DE ALMEIDA & 53 & 16 & PSTU & 84.609 & $\begin{array}{l}\text { NÃO } \\
\text { ELEITO }\end{array}$ & MASCULINO \\
\hline BR & PRESIDENTE & DILMA VANA ROUSSEFF & 63 & 13 & PT & 47.651 .434 & $\begin{array}{l}2^{\circ} \\
\text { TURNO }\end{array}$ & FEMININO \\
\hline BR & PRESIDENTE & $\begin{array}{l}\text { MARIA OSMARINA MARINA DA SILVA VAZ } \\
\text { DE LIMA }\end{array}$ & 52 & 43 & PV & 19.636 .359 & $\begin{array}{l}\text { NÃO } \\
\text { ELEITO }\end{array}$ & FEMININO \\
\hline
\end{tabular}

Resultados de las elecciones generales de presidente (primera vuelta del 2010) - Tribunal Superior Electoral

\begin{tabular}{|l|l|l|l|l|l|l|l|l|}
\hline \hline UF $\uparrow \equiv$ & \multicolumn{1}{|c|}{ Cargo } & \multicolumn{1}{|c|}{ Candidato } & Idade & $\mathrm{Nr}$ & Partido & $\begin{array}{c}\text { Votação } \\
\text { Nominal }\end{array}$ & \multicolumn{1}{c|}{ Situação } & \multicolumn{1}{c|}{ Sexo } \\
\hline $\mathrm{BR}$ & PRESIDENTE & JOSÉ SERRA & 68 & 45 & PSDB & 43.711 .388 & NÃO ELEITO & MASCULINO \\
BR & PRESIDENTE & DILMA VANA ROUSSEFF & 63 & 13 & PT & 55.752 .529 & ELEITO & FEMININO \\
\hline
\end{tabular}

Resultados de las elecciones generales de presidente (segunda vuelta del 2010) - Tribunal Superior Eleitoral 
TIPISMANA ARRIOLA / El auge de la derecha en la crisis posneoliberal en América Latina...

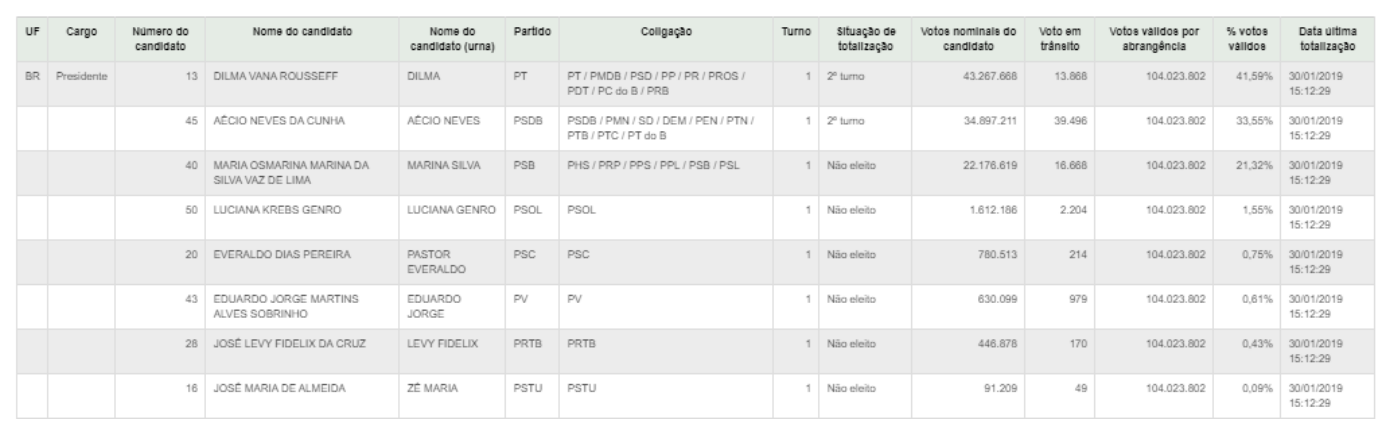

Resultados de las elecciones generales de presidente (primera vuelta del 2014) - Tribunal Superior Eleitoral

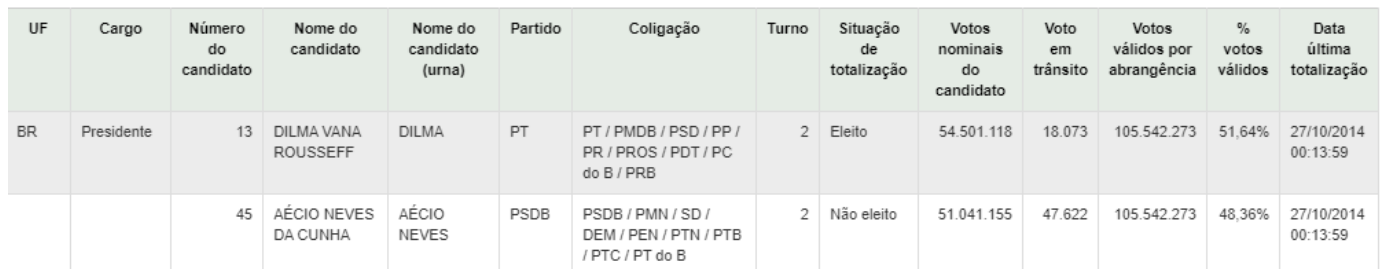

Resultados de las elecciones generales de presidente

(segunda vuelta del 2014) - Tribunal Superior Eleitoral

\begin{tabular}{|c|c|c|c|c|c|c|c|c|c|c|c|c|}
\hline uF & Cargo & $\begin{array}{l}\text { Número do } \\
\text { candidato }\end{array}$ & Nome do candidato & $\begin{array}{c}\text { Nome do } \\
\text { candidato } \\
\text { (urna) }\end{array}$ & Partido & Coligação & Turno & $\begin{array}{l}\text { Situacão de } \\
\text { totalizaçãa }\end{array}$ & $\begin{array}{l}\text { Votos nominais } \\
\text { do candidato }\end{array}$ & $\begin{array}{l}\text { Votos válidos por } \\
\text { abrangência }\end{array}$ & $\begin{array}{l}\text { \%v votos } \\
\text { validos }\end{array}$ & $\begin{array}{l}\text { Data última } \\
\text { totalizaçấo }\end{array}$ \\
\hline \multirow[t]{8}{*}{$B R$} & Presidente & 17 & JAIR MESSIAS BOLSONARO & $\begin{array}{l}\text { JAIR } \\
\text { BOLSONARO }\end{array}$ & PSL & PSL/PRTB & 1 & $2^{\circ}$ turno & 49.277 .010 & 107.050 .749 & $40,03 \%$ & $\begin{array}{l}22 / 012019 \\
14: 12: 19\end{array}$ \\
\hline & & 13 & FERNANDO HADDAD & $\begin{array}{l}\text { FERNANDO } \\
\text { HADDAD }\end{array}$ & PT & PT/PC do B / PROS & 1 & $2^{*}$ turno & 31.342.051 & 107.050 .748 & $29.28 \%$ & $\begin{array}{l}22: 012019 \\
14: 12: 19\end{array}$ \\
\hline & & 12 & CIRO FERREIRA GOMES & CIRO GOMES & PDT & PDT/AVANTE & 1 & Näo eletito & 13.344 .371 & 107.050.749 & $12.47 \%$ & $\begin{array}{l}22 / 01: 2018 \\
14: 12: 19\end{array}$ \\
\hline & & 45 & $\begin{array}{l}\text { GERALDO JOSÉ RODRIGUES } \\
\text { ALCKMIN FILHO }\end{array}$ & $\begin{array}{l}\text { GERALDO } \\
\text { ALCKMN }\end{array}$ & PSDB & $\begin{array}{l}\text { PSDB / PTB / PP / PR/ DEM / } \\
\text { SOLDARIEDADE / PPS / PRB / PSD }\end{array}$ & 1 & Nä̃o elêto & 5.096 .350 & 107.050 .748 & $4,76 \%$ & $\begin{array}{l}22201: 2019 \\
14: 12: 19\end{array}$ \\
\hline & & 30 & $\begin{array}{l}\text { JOÄO DIONISIO FILGUEIRA } \\
\text { BARRETO AMOEDO }\end{array}$ & JOÃO AMOÉDO & Novo & Novo & 1 & Näo elento & 2.679 .745 & 107.050 .748 & $2.50 \%$ & $\begin{array}{l}22 / 101 / 2019 \\
14: 12: 19\end{array}$ \\
\hline & & 51 & $\begin{array}{l}\text { BENEVENUTO DACIOLO } \\
\text { FONSECADOS SANTOS }\end{array}$ & $\begin{array}{l}\text { CABO } \\
\text { DACIOLO }\end{array}$ & PATRI & PATRI & 1 & Nâo elêto & 1.348 .323 & 107.050 .748 & $1.26 \%$ & $\begin{array}{l}222012012019 \\
14: 12: 19\end{array}$ \\
\hline & & 15 & $\begin{array}{l}\text { HENRIOUE DE CAMPOS } \\
\text { MEIRELLES }\end{array}$ & $\begin{array}{l}\text { HENRIOUE } \\
\text { MEIRELLES }\end{array}$ & MOB & MDB/PHS & 1 & Nâo elèto & 1.288 .950 & 107.050 .748 & $1.20 \%$ & $\begin{array}{l}22: 012019 \\
14: 12: 19\end{array}$ \\
\hline & & 18 & $\begin{array}{l}\text { MARIA OSMARINA MARINA DA } \\
\text { SILVA VAZ DE LIMA }\end{array}$ & MARINA SILVA & REDE & REDE / PV & 1 & Nầ elêto & 1.069 .578 & 107.050 .749 & $1.00 \%$ & $\begin{array}{l}22: 012012019 \\
14: 12: 19\end{array}$ \\
\hline
\end{tabular}

Resultados de las elecciones generales de presidente (primera vuelta del 2018) - Tribunal Superior Eleitoral

\begin{tabular}{|c|c|c|c|c|c|c|c|c|c|c|c|c|}
\hline UF & Cargo & $\begin{array}{l}\text { Número do } \\
\text { candidato }\end{array}$ & Nome do candidato & $\begin{array}{c}\text { Nome do } \\
\text { candidato } \\
\text { (Urna) }\end{array}$ & Partido & Coligą̧ão & Turno & $\begin{array}{l}\text { Situação de } \\
\text { totalizą̧ão }\end{array}$ & $\begin{array}{c}\text { Votos } \\
\text { nominais do } \\
\text { candidato }\end{array}$ & $\begin{array}{c}\text { Votos válidos } \\
\text { por } \\
\text { abrangência }\end{array}$ & $\begin{array}{c}\% \\
\text { votos } \\
\text { validos }\end{array}$ & $\begin{array}{l}\text { Data última } \\
\text { totalização }\end{array}$ \\
\hline \multirow[t]{2}{*}{$B R$} & Presidente & 17 & $\begin{array}{l}\text { JAIR MESSIAS } \\
\text { BOLSONARO }\end{array}$ & $\begin{array}{l}\text { JAIR } \\
\text { BOLSONARO }\end{array}$ & PSL & PSL/ PRTB & 2 & Eletio & 57.787 .847 & 104.838 .753 & $55,13 \%$ & $\begin{array}{l}27 / 0222019 \\
18: 15: 58\end{array}$ \\
\hline & & 13 & FERNANDO HADDAD & $\begin{array}{l}\text { FERNANDO } \\
\text { HADDAD }\end{array}$ & PT & PT/PC do B / PROS & 2 & Năo eletito & 47.040 .900 & 104.838 .753 & $44,87 \%$ & $\begin{array}{l}27702 / 2019 \\
18: 15: 58\end{array}$ \\
\hline
\end{tabular}

Resultados de las elecciones generales de presidente

(primera vuelta del 2018) - Tribunal Superior Eleitoral 